\title{
A pilot study to evaluate the effect of increased colonic propionate on appetite during a hypocaloric diet
}

\author{
M. Khatib ${ }^{1}$, E. Chambers ${ }^{1}$, D. Morrison ${ }^{2}$ and G. Frost ${ }^{1}$ \\ ${ }^{1}$ Department of Diabetes, Endocrinology, and Metabolism, Division of Medicine, Imperial College, London W12 0NN, \\ UK and ${ }^{2}$ Scottish Universities Environmental Research Centre (SUERC), University of Glasgow, East Kilbride, \\ Glasgow G75 OQF, UK.
}

Lifestyle interventions based on weight loss diets have highlighted that weight loss increases subjective hunger and may contribute to long-term weight regain ${ }^{(1)}$. The short chain fatty acid (SCFA) propionate stimulates the release of anorectic gut hormones GLP-1 and PYY through activation of free fatty acids receptors 2 and 3 (FFAR2, FFAR3) found on enetroendocrine L-cells in vivo ${ }^{(2)}$ and in vitro ${ }^{(3)}$. Acute supplementation of inulin propionate ester, which delivers propionate to the colon, increased the release of GLP-1 and PYY, and decreased food intake ${ }^{(4)}$. Chronic supplementation of inulin-propionate ester for 24 weeks to overweight adults as part their habitual diet reduced weight gain and decreased visceral fat content ${ }^{(4)}$. The aim of the present study was to investigate the effect of inulin propionate ester supplementation on appetite while following a weight loss diet.

We hypothesized that dietary supplementation with inulin propionate ester combined with a weight loss diet can control appetite and aid with the weight loss.

10 healthy males $(20 \%)$ and females $(80 \%)$ (Mean (SEM), age $33(3)$ years; Body mass index (BMI $\left.\left.28.9(1) \mathrm{kg} / \mathrm{m}^{2}\right)\right) \mathrm{were}$ recruited and completed 12 weeks of intervention in a randomised, controlled, parallel design. Participants received inulin control $(\mathrm{n}=5$; four females) or inulin propionate ester $(n=5$; four females). A hypocaloric diet ( $20 \%$ below estimated daily energy requirements) aimed for approximately $5 \%$ weight loss based on healthy general guidelines and calorie distribution per day. Two study-visits, baseline and after the 12-week supplementation period, were conducted to assess outcome measures. On the study days, and after 12 hours overnight fast, participants attended the clinical research facility to receive a standard breakfast $(358 \mathrm{kcal} ; 61.6 \mathrm{~g} \mathrm{CHO}$, $6.7 \mathrm{~g}$ Fat, $6 \mathrm{~g}$ protein, $9.4 \mathrm{~g}$ fibre). At 240 minutes participants were served an ad libitum meal (246 kcal/100 g; $29.3 \mathrm{~g} \mathrm{CHO,} 11.9 \mathrm{~g}$ protein, $8.5 \mathrm{~g}$ fat, $2.3 \mathrm{~g}$ fibre). Subjective hunger and satiety was monitored with the use of visual analogue scales (VAS). The results demonstrated that participants from both groups lost weight after 12 weeks although this was not significantly different when compared between groups ( $\Delta$ weight $2.6(0.9) \mathrm{kg}$ for inulin propionate ester and $3.0(1.4) \mathrm{kg}$ for the inulin groups respectively; unpaired t-test for the means; $\mathrm{P}=0 \cdot 8)$. Appetite measures were significantly decreased in inulin propionate ester group when compared with baseline. This was clearly shown in composite analogue score (paired t-test for the AUC; $\mathrm{P}=0.01$ ), and when considering specific questions (paired t-test for the AUC; Hunger $\mathrm{P}=0.0001$, Desire to eat $\mathrm{P}=0.02$ ). Postprandial GLP-1 secretion increased following 12 weeks of inulin propionate ester supplementation compared with baseline (paired t-test for the iAUC of 0-60 min; $\mathrm{P}=0.09$ ). The inulin group showed no significant differences in VAS nor GLP-1 results. PYY results showed no differences within nor between the groups.

In conclusion, long term supplementation of inulin propionate ester increases satiety and reduces hunger in humans but this is not through an effect of PYY hormone. This study was completed in accordance with the Declaration of Helsinki (Clinicaltrials.gov (NCT03322514)).

1. Sumithran P, Prendergast L, Delbridge E et al. (2011) new Engl J of Med 365, 1597-604.

2. Psichas A, Sleeth ML, Murphy KG et al. (2015) Int J Obes 39(3), 424-9.

3. Tolhurst G, Heffron H, Lam YS et al. (2012) Diabetes 61(2), 364-71.

4. Chambers ES, Viardot A, Psichas A et al. (2014) Gut 64(11), 1744-54. 